\title{
A Novel Perspective on the Proactive and Reactive Controls of Executive Function in Chronic Stroke Patients.
}

Qiuhua Yu

Sun Yat-sen University First Affiliated Hospital

Xiaomin Huang

Sun Yat-sen University First Affiliated Hospital

Baofeng Zhang

Sun Yat-sen University First Affiliated Hospital

Zhicheng Li

Sun Yat-sen University First Affiliated Hospital

Tao Zhang

Sun Yat-sen University First Affiliated Hospital

Ziwei Hu

Gannan Medical University

Minghui Ding

Sun Yat-sen University First Affiliated Hospital

Zhenwen Liang

Jinan University First Affiliated Hospital

Wai Leung Ambrose Lo ( $\nabla$ ambroselo0726@outlook.com )

Sun Yat-sen University First Affiliated Hospital https://orcid.org/0000-0001-7350-2157

Research

Keywords: executive function, proactive control, reactive control, post-stroke, cognitive decline

Posted Date: January 4th, 2021

DOI: https://doi.org/10.21203/rs.3.rs-136559/v1

License: (c) (1) This work is licensed under a Creative Commons Attribution 4.0 International License. Read Full License

Version of Record: A version of this preprint was published at Frontiers in Neurology on February 28th, 2022. See the published version at https://doi.org/10.3389/fneur.2022.766622. 


\section{Abstract}

Objectives

Executive function is considered to be a main factor that affects the rehabilitation outcome in people with stroke. The proactive and reactive control process required when executing a complex task remains unclear in patients with stroke.

\section{Methods}

Seventeen patients with chronic stroke and seventeen healthy individuals were recruited. Proactive and reactive controls of executive function were assessed the by the task-switching paradigm and the AX version of the Continuous Performance Task (AX-CPT). General executive functions was assessed by the Color Trial Test (CTT) and Stroop Test. The behavioral data of the task-switching paradigm were analyzed by a three-way repeated-measures ANOVA, and the AXCPT data were analyzed by two-way repeated-measures ANOVA.

Results

For efficiency scores in task switching paradigm, trial×group interaction effect was significant $(p=0.006)$. Post hoc analysis on trial×group effect showed no significant between-trial difference in accuracy rate in the stroke group $(p=0.187)$, whereas the accuracy rates in the repeat trial was significantly higher than the switch trial in the control group regardless of $100 \%$ or $50 \%$ validity $(p<0.001)$. Participants in the stroke group had lower accuracy rates than the controls in both repeat $(p=0.002)$ and switch trials $(p=0.068)$. For the AX-CPT, the main effects of condition $(p<0.001)$ and group $(p=0.005)$ on response time were statistically significant. The interaction effect of condition $\times$ group was also significant $(p=0.044)$. Post hoc analysis for condition $\times$ group indicated that the stroke groups had a significantly longer response time in the BX condition than the control group $(p<0.001)$. The stroke cohort showed significantly longer completion time in CTT2 $(p<0.001)$ and larger word interference for completion time in Stroop test $(p=0.029)$ than the control cohort. The response time in BX condition was significant factor in the regression model $(R 2=0.688, B=0.829, p<0.001)$, but not significant in $A Y$ condition ( $p>0.050)$.

\section{Conclusions}

Post-stroke survivors showed deficits in the performance of proactive control but not in the performance of reactive control. Deficits in proactive control may be related to impairment of working memory. Interventions that focus on proactive control may result in improved clinical outcome.

\section{Introduction}

Approximately $50 \%$ to $75 \%$ of chronic post-stroke survivors have mild-to-moderate cognitive dysfunction (Desmond et al., 2000). Executive functions are the higher order of cognitive functions that consist of set-shifting, initiation, monitoring one's behavior and self-regulating functions (Laakso et al., 2019). Basic cognitive functions could be modulated and organized by executive functions to achieve goal-oriented behaviors (Tsuchida \& Fellows, 2013). The research conducted by Laakso et al., (2019) showed that stroke patients had worse performance in executive functions assessments than healthy individuals, including the measures of set-shifting, initiation, response inhibition and strategy formation. Executive function was considered to be the main factor that influenced the clinical outcome and the overall functional status in patients with stroke after rehabilitation (Skidmore et al., 2010). This subsequently contribute to limitations in performing daily activities and participating in social activities (Laakso et al., 2019; Zahuranec, Skolarus, Feng, Freedman, \& Burke, 2017).

Montreal Cognitive Assessment (MoCA) is commonly used as a screening test of executive functions by three subtests, which are Trail Making B task, a phonemic fluency task and a two-item verbal abstraction task (Nasreddine et al., 2005). Executive dysfunctions are indicated if the patient could not complete the subtests. There are others specifically designed assessments to evaluate executive functions such as the Stroop test or the Color Trail Test (CTT) (Laakso et al., 2019). A previous study showed that a large sample of stroke or brain tumor patients with lesion in the frontal region performed worse in the Stroop test than healthy individuals. This suggested impairment of inhibition ability in patients with stroke (Cipolotti et al., 2015). However, MoCA, Stroop test and CTT do not consider the patients' capacity in different executive control processes, e.g., after a cue or target stimulus. Braver (2012) proposed the theory of dual mechanisms of control (DMC) that comprised of proactive and reactive controls. Proactive control is an early selection of goal-relevant information that is processed in a sustained manner of the working memory before the occurrence of the cognitively demanding events (Braver, 2012). It plays an important role in orienting the behavior before the event occurs in our daily life. On the contrary, reactive control refers to the immediate resolution of the current conflict or interference (Braver, 2012), especially when the conflict is without any preparation. The DMC model has been employed to explore the performance of executive function in different sample population. For instance, published literature reported that elderly people and children tended to employ reactive control, while young adults were more rely on proactive control in the response-compatibility task (Friedman, Nessler, Cycowicz, \& Horton, 2009) and in the AX version of Continuous Performance Task (AX-CPT)(Braver, Paxton, Locke, \& Barch, 2009). Thus, the DMC theory provides a better way to understand the flexibility of behavior regulation in complex situations in our daily life (Braver, 2012).

Proactive control impairment could be presented as the inability to employ the information conveyed by the cue to prepare for a response to resolve an upcoming conflict(Braver, 2012). Execution of a functional movement requires an extent of motor anticipation (Kalaska, 2009), which was comparable to that in the proactive control of the DMC model (Yu et al., 2019). The results of a study that utilized electroencephalogram to assess motor anticipation in the patients with stroke indicated that motor anticipation impairment and hand motor function was moderately associated with motor planning (Chen et al., 2018). In Delphine et al.'s study, eight stroke patients were required to grasp and hold a brisk loading under predictive and reactive conditions. The onset time of grip force after the impact was significantly later in the paretic hand of stroke patients than in controls during both predictive and reactive conditions (Dispa, Thonnard, \& Bleyenheuft, 2014). Most of the studies published to date explored the executive dysfunctions without investigating the proactive and reactive controls in patients with chronic stroke. It remains unclear on the behavior regulation involving proactive and reactive controls in patients with chronic stroke when executing a complex task. 
This cross-sectional study aimed to explore the performance of proactive and reactive controls of executive functions in post-stroke survivors by the taskswitching paradigm and AX-CPT. The hypothesis of the present study was that proactive control was more affected than reactive control when performing a complex functional task in patients with stroke.

\section{Methods}

\subsection{Participants}

Participants were recruited from the inpatient unit of the Department of Rehabilitation Medicine, the First Affiliated Hospital of the Sun Yat-sen University. All of the participants were screened by a trained doctor according to the following inclusion and exclusion criteria. Inclusion criteria of the stroke cohort were: (1) age between 40 and 65 years old; (2) three months after stroke occurrence; (3) self-reported or informant-reported memory or cognitive complaints; (4) righthanded. The exclusion criteria of the stroke cohort were: (1)a MoCA score of less than or equal to 14; (2) severe sensorimotor impairment; (3) aphasia identified by the Western Aphasia Battery with aphasia quotient less than 93.8; (4) history of neurological or psychiatrist conditions prior to stroke occurrence; (5) participating in another intervention trial. Healthy participants who had matched age and education level were recruited as the control cohort. Written informed consent was obtained from the participants prior to enrollment. Ethical approval for the present study was granted by the Institutional Ethical Committee for Clinical Research and Animal Trials of the First Affiliated Hospital of Sun Yat-sen University (Approval No.ख[2020]073).

\subsection{Instruments and experiment design}

The task-switching paradigm and AX-CPT were employed to assess the performance of proactive and reactive controls of executive function. The design of task-switching paradigm and AX-CPT made reference to the published studies (Gonthier, Macnamara, Chow, Conway, \& Braver, 2016; Yu et al., 2019). The general executive function was accessed by the CTT and the Stroop test. The task-switching paradigm and AX-CPT were conducted in the software E-prime 2.0 (Psychological Software Tools, Pittsburgh, PA, USA).

\subsubsection{Task-switching paradigm}

In the task-switching paradigm, each trial began with a task cue $(4 \mathrm{~cm} \times 4 \mathrm{~cm})$ at the center of the screen for $1500 \mathrm{~ms}$ (Figure 1$)$. The cue was followed by a target stimulus $(4 \mathrm{~cm} \times 4 \mathrm{~cm})$ that lasted for $2000 \mathrm{~ms}$. Participants were instructed to give a response as soon as possible within the $2000 \mathrm{~ms}$ after the onset of the target stimulus. A blank interval of $1000 \mathrm{~ms}$ then appeared and the next trial began. There were two cue validities: $100 \%$ and $50 \%$. $100 \%$ valid cues were

shown as a solid square ( ) or a diamond ( $50 \%$ valid cue was shown as a solid star (*) . The cue with 100\% validity asked the participants to prepare for the response selection rules based on the cue, whereas the cue with $50 \%$ validity did not convey any information about the following target stimulus. Thus, proactive and reactive controls were manipulated by the $100 \%$ and $50 \%$ validities cue, respectively. The ratio of the two types of cue validities was 1:1. The target stimulus formed by a digit ( 1 or 2 ) appeared inside the shape (a square or diamond) denoted two sets of response selection rules in this paradigm. One response selection rule was that a square with digit " 1 " was for participant to press the button v on the computer keyboard, and square with digit "2" was the button b. The other response selection rule was that the diamond with digit "1" was button b and the diamond with digit " 2 " was button v. In each trial, participants were required to make a response by following a rule that was different from (switch trial) or the same as (repeat trial) the previous trial. In repeat trial the response rule was the same as the previous trial, whereas in switch trial the response rule was different from the previous one. The response time and accuracy rates were recorded during experiment. There were two blocks and 61 trials for each block, in which the first trial was excluded for data analysis. It took around 6 minutes to complete one block followed by a 2-to-3 min break. Mixed block included the same ratio of both repeat (non-switch) trials and switch trials.

\subsubsection{AX-CPT}

AX-CPT included four cue-probe pairs, which were A-X, A-Y, B-X and B-Y.A or B served as a cue, $X$ or $Y$ served as a probe (Figure 2$)$. The cues (4cm $\times 4 \mathrm{~cm})$ and probe stimulus were presented at the center of the screen for $250 \mathrm{~ms}$. A blank screen appeared in an inter-stimulus interval of $1000 \mathrm{~ms}$. After the onset of target stimulus, the participant was asked to give a response as soon as possible within the $1000 \mathrm{~ms}$. Participants were informed to press the button " $v$ " on the keyboard as a target response for the probe " $\mathrm{X}$ " following the cue " $\mathrm{A}$ ", and press the button " $\mathrm{b}$ " as a non-target response for the other three cue-probe pairs. $70 \%$ of the trials were for $A X$ condition, while $10 \%$ of the trials were for each of the other three conditions. The order of these four conditions was randomized. In total, there were two blocks and each block composed of 30 trials. It took approximately 2 minutes to complete one block with a short break between two blocks.

The AX-CPT was designed to assess the proactive control to maintain the cue in the working memory and reactive control to inhibit a prepotent response (Ryman et al., 2019). Proactive control was primarily measured by the BX condition, in which adequate preparation elicited by the cue could enhance the reaction time for the probe. In contrast, reactive control was primarily measured by the AY condition, which required the greatest amount of reactive control to inhibit the prepotent motor response predicted by the cue of " $\mathrm{A}$ ".

\subsubsection{Color Trail Test}


CTT consisted of two parts (CTT1 and CTT2). For CTT1, numbered circles from 1 to 25 were randomly printed on a sheet of paper. Participants were asked to draw a line to join up all the circles in numerical order. CTT2 involved two circles for each number. One circle was in yellow and the other one was in red. Participants were required to draw a line to join up all the numbers from 1 to 25 , alternating between the two colors (Zhou et al., 2014). The time it took for completion, the number sequence and color sequence errors were recorded by the examiner. An interference effect reflecting the shifting function was calculated by the completion time of CCT2 minus the completion time of CTT1 (Interference effect = CTT2 - CTT1).

\subsubsection{Stroop test}

Stroop test was performed to assess the function of conflict inhibition. Stroop test consisted of two sub-tasks, which were naming color and naming color in the printed words. The naming color task required participants to name the color patches printed in red, yellow, green and blue colors. The task of naming color in printed word required participants to name the ink color of a set word, ignoring the words which themselves were the names of other colors. The 100 words or color patches of each sub-task were arranged in a 10×10 matrix. Participants were required to complete the subtasks correctly as quick as they could. The response time it took for completion and the number of errors during each sub-task were recorded. The word interference of completion time, which is the completion time of naming colors minus the completion time of naming colors in printed words, was also included in the data analysis.

\subsection{Procedure}

Background information of age, sex, height, weight, education experience, months after stroke, type of stroke and lesion side and location were recorded from all participants. In task switching paradigm and AX-CPT, the participant was seated in a quiet room in a relaxed position. A laptop computer for displaying the visual stimulus in task switching paradigm and AX-CPT was placed at a distance of $65-75 \mathrm{~cm}$ in front of the participant. The task switching paradigm and AXCPT were randomly assigned to the participants. Before testing, the participant had the practice session to be familiar with each task. After finishing the taskswitching paradigm and $\mathrm{AX}-\mathrm{CPT}$, the participant completed the MoCA, CTT and Stroop test in random order.

\subsection{Data analysis}

Descriptive analysis was conducted to describe the characteristics of the sample populations. Independent t-test was applied to analyze the descriptive characteristics, the behavior data of CTT, Stroop test of the two groups of participants. Chi-square test was conducted to analyze the between-group difference in sex. The behavioral data of the task-switching paradigm were analyzed by a three-way repeated-measures ANOVA: the within-subject factor was validity ( $100 \%$ or $50 \%$ ) and trial (repeat or switch), and the between-subject factor was group (stroke or control group). The data of task switching paradigm showed significant correlations between participants' accuracy rates and reaction times in the control group (repeat trials with $100 \%$ cue validity: $r=-0.693, p=0.002$; switch trials with $100 \%$ cue validity: $r=-0.537, p=0.026$; repeat trials with $50 \%$ cue validity: $r=-0.729, p=0.001$; switch trials with $50 \%$ cue validity: $r=-0.517$, $p=0.033$ ). These significant correlations suggested that a potential accuracy-speed trade-off, which would confound the results. To tackle the problem of accuracy-speed trade-off, the efficiency score (accuracy rate divided by reaction time) was used to analyze the behavioral data in each condition of task switching paradigm (Gonzalez-Valenzuela, Diaz-Giraldez, \& Lopez-Montiel, 2016; Zou, Chau, Ting, \& Chan, 2017). The behavior data of the AX-CPT were analyzed using a two-way repeated-measures ANOVA: the within-subject factor was condition (AY or BX), and the between-subject factor was group (stroke or control group). The dependent variables were the response time and accuracy rates in the AX-CPT and the response times, accuracy rates and efficiency scores in repeat and switch trials in the task-switching paradigm. Pearson's correlation analysis was conducted to test the relationship between the general executive functions and the performance in task-switching paradigm and AX-CPT of the two cohorts. If multiple variables in task-switching paradigm or AX-

CPT were significantly related to general executive functions, multiple regression model with stepwise method was then conducted to verify the contributions of each variable to the capacity of general executive functions. All analyses were performed using the SPSS 20.0 (IBM SPSS Inc. Chicago, IL, USA) Windows software. The level of significance in the multivariate analyses was set at $p 0.05$.

\section{Results}

\subsection{Participants}

Seventeen participants with stroke (12 males and 5 females) and 17 control participants (8 males and 9 females) were recruited in this study. The sample characteristics of the two cohorts are presented in Table 1. The between-group difference in sex was not significant $(p=0.296)$. No significant between-group difference was shown in age, weight, height, BMI, and education experience $(p>0.050)$. The MoCA score in the stroke group was significantly lower than the control group ( $p \otimes 0.001)$. Table 2 presents the clinical characteristics of each participant in the stroke cohort.

Table 1: Descriptive characteristics of the two groups of participants. 


\begin{tabular}{|c|c|c|c|c|}
\hline & $\begin{array}{l}\text { Stroke group } \\
\text { Mean(SD) }\end{array}$ & $\begin{array}{l}\text { Control group } \\
\text { Mean (SD) }\end{array}$ & $\mathrm{t}$ & $p$ \\
\hline Age (years) & $53.82(7.74)$ & $52.18(7.09)$ & 0.647 & 0.522 \\
\hline Weight $(\mathrm{kg})$ & $63.41(9.03)$ & $59.65(10.93)$ & 1.093 & 0.283 \\
\hline Height (m) & $1.67(0.05)$ & $1.63(0.08)$ & 1.443 & 0.159 \\
\hline BMI & $20.82(3.16)$ & $22.22(3.21)$ & -1.280 & 0.210 \\
\hline Education experience (years) & $11.53(2.74)$ & $11.65(3.10)$ & -0.117 & 0.907 \\
\hline MoCA & $22.12(2.78)$ & $26.82(1.55)$ & -6.093 & $\bowtie 0.001$ \\
\hline
\end{tabular}

Table 2: The clinical characteristics of the 17 chronic post-stroke participants. 


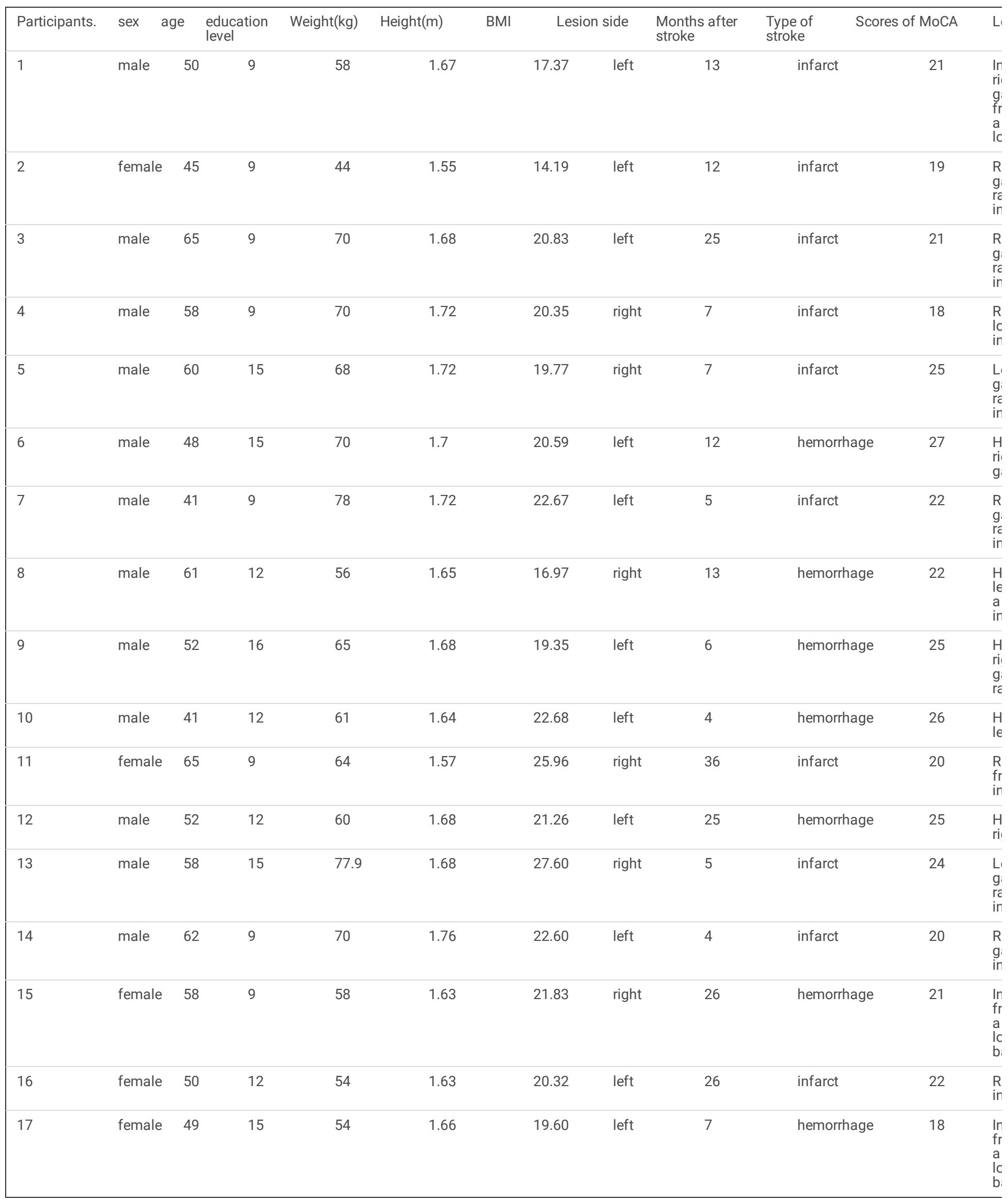

\subsection{Task-switching paradigm}


Participants' accuracy rates of the repeat and switch trials in task-switching paradigm are presented in Table 3 . The main effects of validity $[F(1,32)=1.319$, $\left.p=0.259, n p^{2}=0.040\right]$ and trial $\left[F(1,32)=1.315, p=0.260, n p^{2}=0.039\right]$ were not statistically significant. However, the group main effect was statistically significant $\left[F(1,32)=12.174, p=0.001, \eta p^{2}=0.276\right]$. The interaction effect of validity $\times$ trial $\times$ group was not significant $\left[F(1,32)=0.429, p=0.517, \eta p^{2}=0.013\right]$. Significant group effect suggested that participants in the stroke group had lower accuracy rates than the controls in both validities and trials.

Participants' response time of repeat and switch trials in task-switching paradigm are shown in Table 3 . The main effects of validity $[F(1,32)=12.508$, $\left.p=0.001, \eta p^{2}=0.281\right]$ and trial $\left[F(1,32)=36.513, p<0.001, \eta p^{2}=0.533\right]$ were significant. However, the group effect was not significant $[F(1,32)=0.097, p=0.758$, $\left.\eta \mathrm{p}^{2}=0.003\right]$. The interaction effect of validity $\times$ trial $\times$ group was not significant $\left[F(1,32)=0.1 .186, p=0.284, \eta p^{2}=0.036\right]$. The interaction effect of trial $\times$ group was also not significant $\left[F(1,32)=1.328, p=0.258, n p^{2}=0.040\right]$.

Participants' efficiency scores (accuracy rate divided by reaction time) of repeat and switch trials in task-switching paradigm are shown in Table 4 . The main effects of validity $\left[F(1,32)=1.444, p=0.238, n p^{2}=0.043\right]$ were not statistically significant. However, the main effects of trial $[F(1,32)=23.352, p<0.001$, $\left.\eta \mathrm{p}^{2}=0.422\right]$ and group were statistically significant $\left[F(1,32)=8.181, p=0.007, \eta p^{2}=0.204\right]$. The interaction effect of validity $\times$ trial $\times$ group was not significant $\left[F(1,32)=0.014, p=0.906, \eta p^{2}<0.001\right]$, whereas the trial $\times$ group interaction effect was significant $\left[F(1,32)=8.546, p=0.006, \eta p^{2}=0.211\right]$. Post hoc analysis on trial×group effect showed that no significant between-trial difference in efficiency scores in the stroke group $(p=0.187)$, whereas the efficiency scores in the repeat trial were significantly higher than the switch trial in the control group regardless of $100 \%$ or $50 \%$ validity $(p<0.001)$. Participants in the stroke group had lower efficiency scores than the controls in both repeat $(p=0.002)$ and switch trials $(p=0.068)$. These findings also suggested that the stroke group did not perform worse than the control group in the proactive and reactive controls of switching functions.

Table 3: Participants' accuracy rates (\%) and response times (ms) of repeat and switch trials in task-switching paradigm stratified by Group and Validity.

\begin{tabular}{|llllll|}
\hline validity & trial & \multicolumn{2}{l}{ Stroke group } & \multicolumn{2}{l|}{ Control group } \\
\cline { 3 - 6 } & & Mean(SD) & & Mean(SD) & \\
& & ACC & RT & ACC & RT \\
\hline $100 \%$ & repeat & $64.62(21.74)$ & $960.16(160.42)$ & $86.52(14.37)$ & $881.69(139.55)$ \\
\hline & switch & $58.79(21.72)$ & $1084.89(259.57)$ & $75.40(18.75)$ & $1103.36(202.31)$ \\
\hline $50 \%$ & repeat & $59.34(22.43)$ & $1003.37(226.86)$ & $83.87(17.45)$ & $981.95(163.93)$ \\
\hline & switch & $68.24(17.41)$ & $1151.29(244.53)$ & $83.53(14.55)$ & $1161.43(153.97)$ \\
\hline
\end{tabular}

NOTE: $100 \%=100 \%$ valid cue; $50 \%=50 \%$ valid cue; $\mathrm{ACC}=$ accuracy rate; $\mathrm{RT}=$ response time.

Table 4: Participants' efficiency scores (\%/ms) of repeat and switch trials in task-switching paradigm stratified by Group and Validity.

\begin{tabular}{|llll|}
\hline & & Stroke group & Control group \\
\hline validity & trial & Mean(sd) & Mean(sd) \\
\hline $100 \%$ & repeat & $0.069(0.027)$ & $0.103(0.032)$ \\
& switch & $0.057(0.022)$ & $0.073(0.030)$ \\
\hline $50 \%$ & repeat & $0.062(0.027)$ & $0.089(0.025)$ \\
& switch & $0.063(0.023)$ & $0.074(0.018)$ \\
\hline
\end{tabular}

NOTE: $100 \%=100 \%$ valid cue; $50 \%=50 \%$ valid cue.

\subsection{AX-CPT}

The main effect of condition on accuracy rates was significant $\left[F(1,32)=8.196, p=0.007, n p^{2}=0.204\right]$. However, the group effect was not significant $[F(1,32)$ $\left.=3.344, p=0.077, \eta p^{2}=0.095\right]$. The interaction effect of condition $\times$ group was also not significant $\left[F(1,32)=0.719, p=0.403, \eta p^{2}=0.022\right]$.

The main effects of condition and group on response time were statistically significant, $\left[F(1,32)=61.151, p<0.001, \eta p^{2}=0.656\right],[F(1,32)=8.899, p=0.005$, $\left.\eta \mathrm{p}^{2}=0.218\right]$. The interaction effect of condition $\times$ group was also significant $\left[F(1,32)=4.414, p=0.044, \eta p^{2}=0.121\right]$ (Figure 3). Post hoc analysis for condition×group indicated that the stroke participants had a significantly longer response time in the BX condition than control group ( $p<0.001$ ) (Figure 3 ). No significant between-group difference in response time was found in AY condition ( $p=0.169)$. These findings suggested that the proactive control that were related to working memory was impaired in patients with stroke.

\subsection{Color Trail Test}

No significant between-group difference was observed in the error rates of CTT1 and CTT2 ( $p>0.050)$. However, participants in the stroke group showed significantly longer completion time in CTT1 ( $p=0.002)$, CTT2 ( $p<0.001)$, and larger interference effect $(p=0.019)$ than the control group (Table 5).

Page $7 / 13$ 
Table 5: The error rates and response times in the Color Trail Test.

\begin{tabular}{|lllll|}
\hline & $\begin{array}{l}\text { Stroke group } \\
\text { Mean(SD) }\end{array}$ & $\begin{array}{l}\text { Control group } \\
\text { Mean(SD) }\end{array}$ & & $p$ \\
\hline CTT1 NER(\%) & $1.65(3.76)$ & $1.18(2.74)$ & .417 & 0.679 \\
\hline CTT2 NER(\%) & $0.24(0.97)$ & $0.47(1.94)$ & -0.447 & 0.658 \\
\hline CTT2 CER(\%) & $0.71(2.11)$ & $0.00(0.00)$ & 1.376 & 0.188 \\
\hline CTT1 CT(s) & $127.71(68.54)$ & $65.24(18.28)$ & 3.631 & 0.002 \\
\hline CTT2 CT(s) & $179.18(65.33)$ & $90.06(24.17)$ & 5.275 & $<0.001$ \\
\hline interference effect & $51.47(41.52)$ & $24.82(15.87)$ & 2.472 & 0.019 \\
\hline
\end{tabular}

NOTE: NER=number sequence error rates, $\mathrm{CER}=$ color sequence error rates, $\mathrm{CT}=$ completion time.

\subsection{Stroop test}

The error rates and the self-corrected error rates showed no significant between-group difference in naming then color and naming color of the printed word of the Stroop test $(p>0.050)$. However, the stroke group showed significantly longer completion time in naming the color $(p=0.002)$, naming the color of the printed word $(p=0.001)$, and the word interference of CT $(p=0.029)$ (Table 6$)$.

Table 6: The error rates and response times in the Stroop test.

\begin{tabular}{|lllll|}
\hline & $\begin{array}{l}\text { Stroke group } \\
\text { Mean (SD) }\end{array}$ & $\begin{array}{l}\text { Control group } \\
\text { Mean (SD) }\end{array}$ & t & $p$ \\
\hline ER in naming colors (\%) & $1.35(2.06)$ & $0.41(0.94)$ & 1.714 & 0.100 \\
ER in naming colors in & $3.82(5.99)$ & $1.24(2.17)$ & 1.675 & 0.104 \\
printed words (\%) & & & & \\
\hline CT in naming colors (\%) & $125.88(49.81)$ & $82.35(18.09)$ & 3.387 & 0.002 \\
\hline CT in naming colors in & $217.06(65.21)$ & $133.06(68.76)$ & 3.655 & 0.001 \\
printed words (\%) & & & & \\
\hline Word interference of CT & $91.18(38.09)$ & $50.71(62.07)$ & 2.291 & 0.029 \\
\hline
\end{tabular}

NOTE: ER=error rates, SER=self-corrected error rates, $\mathrm{CT}=$ completion time.

\subsection{The relationship between general executive functions, proactive and reactive controls in the two cohorts}

The relationship between general executive functions and proactive and reactive controls of the two groups are presented in Table 7. In the task-switching paradigm, the stroke group showed negative correlations between the accuracy rates of any trial type under $100 \%$ and $50 \%$ validities and the interference effect of CTT ( $r=-0,494 \sim-0.507, p<0.050)$. Thus, the accuracy rates of repeat and switch trials under both $100 \%$ and $50 \%$ validities were put into the multiple regression model as the independent variables for stroke group. The results of multiple regression analysis showed that only the accuracy rate in switch trial under the $50 \%$ validity condition was a significant factor $\left(\mathrm{R}^{2}=0.326, \mathrm{~B}=-0.571, p=0.017\right)$. However, the accuracy rate in switch trial under the $50 \%$ validity was only correlated with the interference effect in CTT in the control group ( $r=-0.614, p=0.009)$. No significant association was found between the task-switching paradigm and the word interference in the Stroop test $(p>0.050)$.

In the AX-CPT, there was no significant correlation with the interference effect in CTT ( $p>0.050)$. Significant correlations were found between the word interference of Stroop test and the response time in $\mathrm{AY}(\mathrm{r}=0.675, p=0.003)$ and $\mathrm{BX}(\mathrm{r}=0.829, p<0.001)$ conditions in the control group but not in stroke group. Thus, the response time of $A Y$ and $B X$ conditions were put into the multiple regression model as the independent variables for the control group. The results of multiple regression analysis showed that only the response time in $B X$ condition was a significant factor $\left(R^{2}=0.688, B=0.829, p<0.001\right)$, whereas the response time in AY condition was not significant $(p=0.433)$. These results further confirmed that stroke patients had impaired proactive control, which was related to conflict monitoring.

\section{Table 7: The relationship between the general executive functions and the performance in task-switching paradigm and AX-CPT of the two groups of participants.}




\begin{tabular}{|c|c|c|c|c|}
\hline & \multicolumn{2}{|c|}{ Interference effect of CTT } & \multicolumn{2}{|c|}{ Word interference of CT of Stroop test } \\
\hline & Stroke & Control & Stroke & Control \\
\hline 100\%_R_ACC & $-.494^{\star}$ & -.213 & -.219 & .016 \\
\hline 100\%_S_ACC & $-.497 *$ & .005 & -.195 & -.172 \\
\hline 50\%_R_ACC & $-.507 *$ & -.191 & -.303 & .188 \\
\hline 50\%_S_ACC & $-.571 *$ & $-.614 * \star$ & -.420 & .335 \\
\hline 100\%_R_RT & .071 & .155 & .314 & .172 \\
\hline $100 \%$ S_RT & -.132 & .267 & -.278 & -.206 \\
\hline 50\%_R_RT & -.079 & .267 & .235 & -.117 \\
\hline 50\%_S_RT & .130 & .045 & -.008 & .025 \\
\hline AY_ACC & .028 & .098 & .279 & -.189 \\
\hline BX_ACC & -.130 & .175 & -.430 & -.133 \\
\hline AY_RT & -.035 & -.403 & .347 & $.675^{\star \star}$ \\
\hline BX_RT & .206 & -.179 & .347 & $.829 * \star$ \\
\hline
\end{tabular}

Note: $100 \%$ denotes $100 \%$ validity; $50 \%$ denotes $50 \%$ validity; $\mathrm{R}=$ repeat; $\mathrm{S}=$ switch; $\mathrm{ACC}=$ accuracy rates; $\mathrm{RT}=$ response time; $\mathrm{CT}=$ completion time. ${ }^{\star}$ denotes $p<$ 0.05. ** denotes $p<0.01$

\section{Discussion}

The present study investigated the performance of proactive and reactive controls of executive functions in post-stroke survivors by the dual cognitive control model. The main findings indicated that post-stroke participants require longer response time in BX condition of the AX-CPT, but did not exhibit a reduction in the proactive and reactive control of switching compared with controls during the task-switching paradigm. These novel findings supported the hypothesis that proactive control was more affected than reactive control when performing a complex functional task.

In BX condition, participants needed to maintain the "B" cue in the working memory, so that adequate preparation which could enhance the reaction time for the probe (Ryman et al., 2019). This study found that the post-stroke participants required longer response time in BX condition of the AX-CPT, suggesting that proactive control was impaired in post-stroke survivors. This finding was supported by two other previous studies that explored the proactive and reactive controls without involving executive functions in the stroke patients (Dispa et al., 2014; Jo et al., 2016). One study showed impairment in the feed-forward adjustments of the synergies prior to a quick action in patients after a single cortical stroke with mild motor impairments (Jo et al., 2016). Dispa et al found that the onset time of grip force after the impact was significantly later in the paretic hand of stroke patients than in controls in both predictive and reactive conditions (Dispa et al., 2014). Proactive control in AX-CPT was reported to be relied upon the anticipation and prevention of interference before it occurred in the working memory (Braver, 2012; Ryman et al., 2019). Several studies have indicated working memory deficits in patients with stroke (Povroznik, Ozga, Vonder Haar, \& Engler-Chiurazzi, 2018; van Geldorp, Kessels, \& Hendriks, 2013). In the study by van Geldorp et al. (2013), the participants were required to remember which cue was presented after a short temporal delay in a computerized delayed-match-to-sample task which included spatial, object, or binding (spatial + object) cues. Compared to healthy controls, the stroke participants showed working memory deficits in the conditions of spatial and object cues. Another study conducted by Roussel et al. (2016) indicated that in post-stroke participants, the dysexecutive profile was characterized by prominent impairments of motor initiation and generation with anticipation loss in the behavioral domain. Thus, in the present study, the worse performance in proactive control observed in the stroke group maybe resulted from the impairment of working memory and anticipation loss.

According to the results of the task-switching paradigm, the stroke group had lower accuracy rates and efficiency scores in both repeat and switch trials than the control group. The two cohorts, however, did not show between-trial difference in $100 \%$ and $50 \%$ validities, which may suggest participants in the stroke group did not perform worse than healthy participants in the proactive and reactive controls of set-shifting function. These findings were consistent with the previous study which demonstrated patients with stroke had different switch costs of accuracy rates compared with the control group in alternating switch task, but not in the cued task-switching (Pohl et al., 2007). The findings in Pohl et al.'s study suggested that task switching in alternating switch task with high demand of endogenous proactive control was impaired in adults after stroke, whereas task switching in the cued task-switching requiring exogenous proactive control was not impaired. The first reason was likely due to the higher difficulty level under endogenous proactive control than under exogenous proactive control. The second reason may be that the key neural structures that are responsible for the proactive control in task switching include the frontal and/or parietal cortex cortices (Ryman et al., 2019; Yu et al., 2019). However, the present study and Pohl et al.'s study did not consider the different lesion locations between the two cohorts. This might potentially contribute to the lack of observed difference between the proactive and reactive controls of switching function in the stroke group.

The efficiency scores in task switching paradigm showed no significant between-trial difference in the stroke group, whereas the efficiency scores in the repeat trial of the control group were significantly higher than those in the switch trial regardless of $100 \%$ or $50 \%$ validity. These findings suggested that post-stroke survivors was less susceptible to proactive interference, which may be due to their lower working memory function. As Allport and colleagues assumed that switch costs arose from proactive interference (G. Wylie \& Allport, 2000). Higher susceptibility of proactive interference in task switching was associated with 
higher working memory capacity (Pettigrew \& Martin, 2016; Steinwascher \& Meiser, 2016). Several studies had indicated working memory deficits in poststroke survivors (Roussel, Dujardin, Henon, \& Godefroy, 2012; van Geldorp et al., 2013), which was likely to be a factor that contribute to lower susceptibility to proactive interference in post-stroke survivors than healthy individuals. Impaired proactive control in the AX-CPT of stroke patients also supported deficits in the working memory since proactive control in the AX-CPT was related to working memory (Ryman et al., 2019).

Larger interference effect in CTT and higher word interference of completion time in Stroop test were observed in stroke participants when compared with healthy controls. The interference effect in CTT assessed the shifting function (Jo et al., 2016), while the word interference of completion time in the Stroop test taps on the conflict inhibition during naming color(Cheng et al., 2012). The results of the CTT and Stroop test suggested that the general executive function was impaired in stroke participants. These findings were consistent with a previous study (Laakso et al., 2019), which showed that stroke patients was performed poorly in the Trail Making test and Stroop test. The results of the correlation and regression analysis between the Stroop test and the performance in BX condition of AX-CPT of the two groups further supported the notion that the proactive control for working memory function was impaired in post-stroke survivors. The process of interference control in the Stroop test include the top-down control process (Popov et al., 2018), which was also required during proactive control in AX-CPT (Manard, Francois, Phillips, Salmon, \& Collette, 2017). These two previous studies reported that prefrontal cortex was the key node activated in the top-down control process in Stroop test and proactive control. Thus, the response time in BX condition related to proactive control was a significant factor related to the performance in Stroop test.

\section{Limitations}

There are several limitations in the present study which limit the interpretation of the data. The sample size was not power calculated and was likely to contain type II error. This might contribute to the lack of between-group difference in the task-switching paradigm. The effect of lesion locations or lesion size on the proactive and reactive controls were not considered in the present study. Future study should consider recruiting sample group that has homogeneous lesion site and size. In addition to behavioral outcomes, future research could adopt electrophysiology and brain-imaging techniques to investigate the underlying neural mechanisms (Redick, 2014). Last but not least, other research results showed a significant gender difference in proactive and reactive controls (Smittenaar et al., 2015). Further studies are required to clarify the gender effect on the performance of proactive and reactive controls in post-stroke survivors.

\section{Conclusion}

Post-stroke survivors showed deficits in the performance of proactive control that are related to working memory. However, post-stroke patients seemed not to show significant deficit in the reactive control of executive functions. Intervention that focus on proactive control may result in improved clinical outcome.

\section{Declarations}

\section{Ethics approval and consent to participate}

Ethical approval for the present study was granted by the Institutional Ethical Committee for Clinical Research and Animal Trials of the First Affiliated Hospital of Sun Yat-sen University (Approval No.『[2020]073). Written informed consent was obtained from the participants prior to enrollment.

\section{Consent for publication}

NA

\section{Availability of data and materials}

Data is available upon request from the corresponding author.

\section{Competing interests}

The authors declared no conflict of interest associated with the work presented in this manuscript.

\section{Funding}

This research project was supported by the National Natural Science Foundation of China (grant number囚81971224, 82002375); Science and Technology Program of Guangzhou (grant number 201803010083) 『and Medical Scientific Research Foundation of Guangdong Province of China (grant number》 A2019452).

\section{Authors' contributions}

All authors have read and approved the final manuscript. All authors meet the four primary ICMJE criteria for authorship. In addition, all authors have been actively involved in the study in different capacities: QY and WLAL designed the study and conducted all stages of the study including data collection, analysis, interpretation, and drafting of the manuscript. XH, HZ, BZ, ZL participated in the design of the research protocol, data analysis and drafting of the manuscript. TZ, ZH, MD, ZL participated in data collection, analysis and interpretation of the data. WLAL and ZL revised the manuscript, interpreted the data and managed the trial.

\section{Acknowledgements}

We would like to thank all the participants who took part in the study and the clinical supporting staff of The First Affiliated Hospital, Sun Yat-sen University. 


\section{References}

Braver, T. S. (2012). The variable nature of cognitive control: a dual mechanisms framework. Trends Cogn Sci, 16(2), 106-113. doi:10.1016/j.tics.2011.12.010

Braver, T. S., Paxton, J. L., Locke, H. S., \& Barch, D. M. (2009). Flexible neural mechanisms of cognitive control within human prefrontal cortex. Proc Natl Acad Sci U S A, 106(18), 7351-7356. doi:10.1073/pnas.0808187106

Chen, L., Mao, Y., Ding, M., Li, L., Leng, Y., Zhao, J., . . Lo, W. L. A. (2018). Assessing the Relationship Between Motor Anticipation and Cortical Excitability in Subacute Stroke Patients With Movement-Related Potentials. Front Neurol, 9, 881. doi:10.3389/fneur.2018.00881

Cheng, Y., Wu, W., Feng, W., Wang, J., Chen, Y., Shen, Y., . . Li, C. (2012). The effects of multi-domain versus single-domain cognitive training in non-demented older people: a randomized controlled trial. BMC Med, 10, 30. doi:10.1186/1741-7015-10-30

Cipolotti, L., Healy, C., Chan, E., MacPherson, S. E., White, M., Woollett, K., .. Shallice, T. (2015). The effect of age on cognitive performance of frontal patients. Neuropsychologia, 75, 233-241. doi:10.1016/j.neuropsychologia.2015.06.011

Desmond, D. W., Moroney, J. T., Paik, M. C., Sano, M., Mohr, J. P., Aboumatar, S., . . Stern, Y. (2000). Frequency and clinical determinants of dementia after ischemic stroke. Neurology, 54(5), 1124-1131. doi:10.1212/wnl.54.5.1124

Dispa, D., Thonnard, J. L., \& Bleyenheuft, Y. (2014). Impaired predictive and reactive control of precision grip in chronic stroke patients. Int J Rehabil Res, 37(2), 130-137. doi:10.1097/MRR.0000000000000045

Friedman, D., Nessler, D., Cycowicz, Y. M., \& Horton, C. (2009). Development of and change in cognitive control: a comparison of children, young adults, and older adults. Cogn Affect Behav Neurosci, 9(1), 91-102. doi:10.3758/CABN.9.1.91

Gonthier, C., Macnamara, B. N., Chow, M., Conway, A. R., \& Braver, T. S. (2016). Inducing Proactive Control Shifts in the AX-CPT. Front Psychol, 7, 1822. doi:10.3389/fpsyg.2016.01822

Gonzalez-Valenzuela, M. J., Diaz-Giraldez, F., \& Lopez-Montiel, M. D. (2016). Cognitive Predictors of Word and Pseudoword Reading in Spanish First-Grade Children. Front Psychol, 7, 774. doi:10.3389/fpsyg.2016.00774

Jo, H. J., Maenza, C., Good, D. C., Huang, X., Park, J., Sainburg, R. L., \& Latash, M. L. (2016). Effects of unilateral stroke on multi-finger synergies and their feedforward adjustments. Neuroscience, 319, 194-205. doi:10.1016/j.neuroscience.2016.01.054

Kalaska, J. F. (2009). From intention to action: motor cortex and the control of reaching movements. Adv Exp Med Biol, 629, 139-178. doi:10.1007/978-0-38777064-2_8

Laakso, H. M., Hietanen, M., Melkas, S., Sibolt, G., Curtze, S., Virta, M., ... Jokinen, H. (2019). Executive function subdomains are associated with post-stroke functional outcome and permanent institutionalization. Eur J Neurol, 26(3), 546-552. doi:10.1111/ene.13854

Manard, M., Francois, S., Phillips, C., Salmon, E., \& Collette, F. (2017). The neural bases of proactive and reactive control processes in normal aging. Behav Brain Res, 320, 504-516. doi:10.1016/j.bbr.2016.10.026

Nasreddine, Z. S., Phillips, N. A., Bedirian, V., Charbonneau, S., Whitehead, V., Collin, I., . . Chertkow, H. (2005). The Montreal Cognitive Assessment, MoCA: a brief screening tool for mild cognitive impairment. J Am Geriatr Soc, 53(4), 695-699. doi:10.1111/j.1532-5415.2005.53221.x

Pettigrew, C., \& Martin, R. C. (2016). The role of working memory capacity and interference resolution mechanisms in task switching. Q J Exp Psychol (Hove), 69(12), 2431-2451. doi:10.1080/17470218.2015.1121282

Pohl, P. S., McDowd, J. M., Filion, D., Richards, L. G., Stiers, W., \& Kluding, P. (2007). Task switching after stroke. Phys Ther, 87(1), 66-73. doi:10.2522/ptj.20060093

Popov, T., Westner, B. U., Silton, R. L., Sass, S. M., Spielberg, J. M., Rockstroh, B., . . Miller, G. A. (2018). Time Course of Brain Network Reconfiguration Supporting Inhibitory Control. J Neurosci, 38(18), 4348-4356. doi:10.1523/JNEUROSCI.2639-17.2018

Povroznik, J. M., Ozga, J. E., Vonder Haar, C., \& Engler-Chiurazzi, E. B. (2018). Executive (dys)function after stroke: special considerations for behavioral pharmacology. Behav Pharmacol, 29(7), 638-653. doi:10.1097/FBP.0000000000000432

Redick, T. S. (2014). Cognitive control in context: working memory capacity and proactive control. Acta Psychol (Amst), 145, 1-9.

doi:10.1016/j.actpsy.2013.10.010

Roussel, M., Dujardin, K., Henon, H., \& Godefroy, O. (2012). Is the frontal dysexecutive syndrome due to a working memory deficit? Evidence from patients with stroke. Brain, 135(Pt 7), 2192-2201. doi:10.1093/brain/aws132

Roussel, M., Martinaud, O., Henon, H., Vercelletto, M., Bindschadler, C., Joseph, P. A., . . Group, G. S. (2016). The Behavioral and Cognitive Executive Disorders of Stroke: The GREFEX Study. PLoS One, 11(1), e0147602. doi:10.1371/journal.pone.0147602 
Ryman, S. G., El Shaikh, A. A., Shaff, N. A., Hanlon, F. M., Dodd, A. B., Wertz, C. J., . . Mayer, A. R. (2019). Proactive and reactive cognitive control rely on flexible use of the ventrolateral prefrontal cortex. Hum Brain Mapp, 4O(3), 955-966. doi:10.1002/hbm.24424

Skidmore, E. R., Whyte, E. M., Holm, M. B., Becker, J. T., Butters, M. A., Dew, M. A., . . Lenze, E. J. (2010). Cognitive and affective predictors of rehabilitation participation after stroke. Arch Phys Med Rehabil, 91(2), 203-207. doi:10.1016/j.apmr.2009.10.026

Smittenaar, P., Rutledge, R. B., Zeidman, P., Adams, R. A., Brown, H., Lewis, G., \& Dolan, R. J. (2015). Proactive and Reactive Response Inhibition across the Lifespan. PLoS One, 10(10), e0140383. doi:10.1371/journal.pone.0140383

Steinwascher, M. A., \& Meiser, T. (2016). How a high working memory capacity can increase proactive interference. Conscious Cogn, 44, $130-145$. doi:10.1016/j.concog.2016.07.002

Tsuchida, A., \& Fellows, L. K. (2013). Are core component processes of executive function dissociable within the frontal lobes? Evidence from humans with focal prefrontal damage. Cortex, 49(7), 1790-1800. doi:10.1016/j.cortex.2012.10.014

van Geldorp, B., Kessels, R. P., \& Hendriks, M. P. (2013). Single-item and associative working memory in stroke patients. Behav Neurol, $26(3), 199-201$. doi:10.3233/BEN-2012-129010

Wylie, G., \& Allport, A. (2000). Task switching and the measurement of "switch costs". Psychol Res, 63(3-4), 212-233. doi:10.1007/s004269900003

Wylie, S. A., Ridderinkhof, K. R., Bashore, T. R., \& van den Wildenberg, W. P. (2010). The effect of Parkinson's disease on the dynamics of on-line and proactive cognitive control during action selection. J Cogn Neurosci, 22(9), 2058-2073. doi:10.1162/jocn.2009.21326

Yu, Q., Chau, B. K. H., Lam, B. Y. H., Wong, A. W. K., Peng, J., \& Chan, C. C. H. (2019). Neural Processes of Proactive and Reactive Controls Modulated by MotorSkill Experiences. Front Hum Neurosci, 13, 404. doi:10.3389/fnhum.2019.00404

Zahuranec, D. B., Skolarus, L. E., Feng, C., Freedman, V. A., \& Burke, J. F. (2017). Activity limitations and subjective well-being after stroke. Neurology, 89(9), 944950. doi:10.1212/WNL.0000000000004286

Zhou, F. C., Hou, W. M., Wang, C. Y., Ungvari, G. S., Chiu, H. F., Correll, C. U., . . Xiang, Y. T. (2014). Prospective memory performance in non-psychotic firstdegree relatives of patients with schizophrenia: a controlled study. PLoS One, 9(11), e111562. doi:10.1371/journal.pone.0111562

Zou, Z., Chau, B. K. H., Ting, K. H., \& Chan, C. C. H. (2017). Aging Effect on Audiovisual Integrative Processing in Spatial Discrimination Task. Front Aging Neurosci, 9, 374. doi:10.3389/fnagi.2017.00374

\section{Figures}

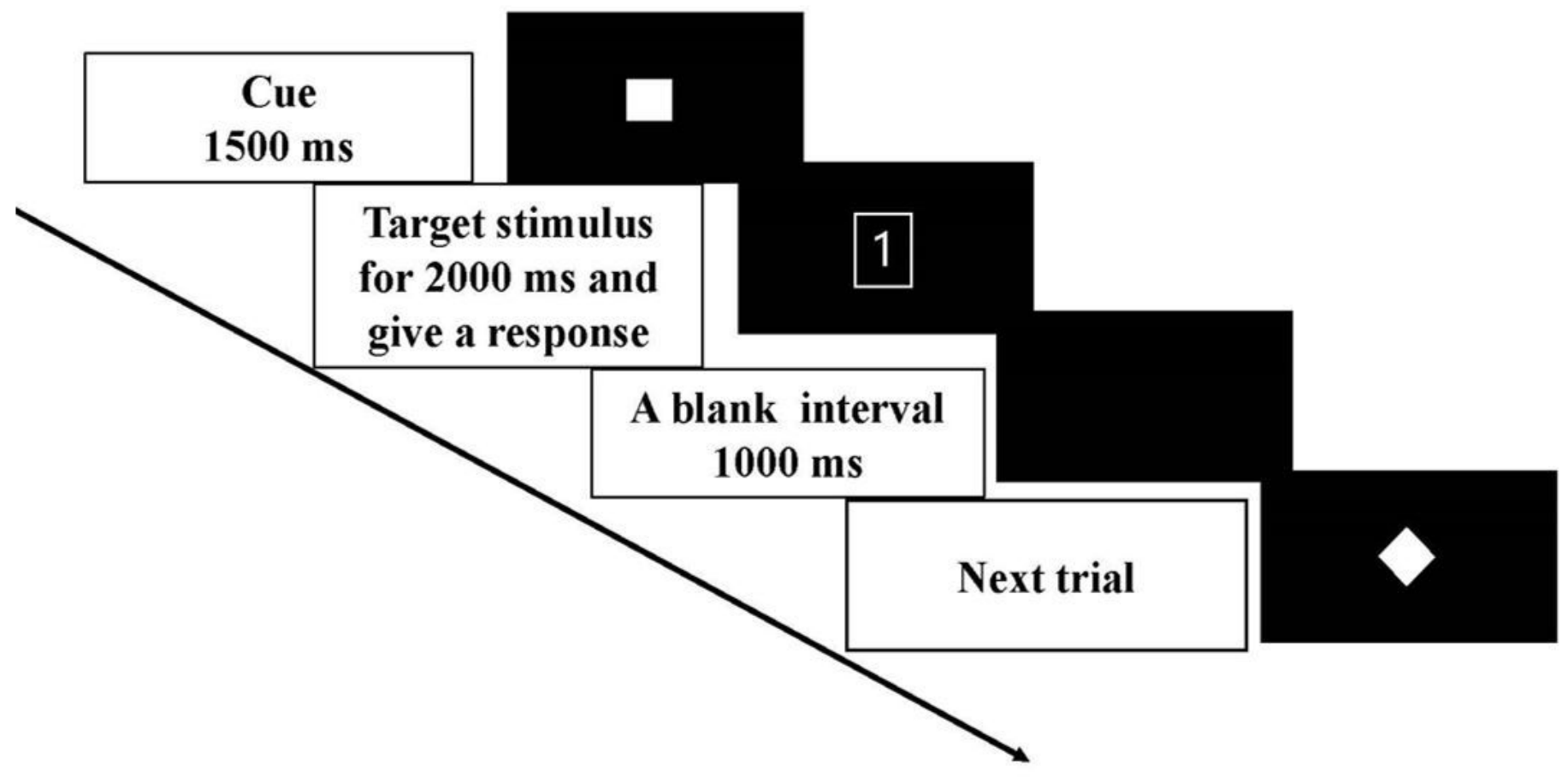

Figure 1

Schematic illustration of time course of one trial in task-switching paradigm. 


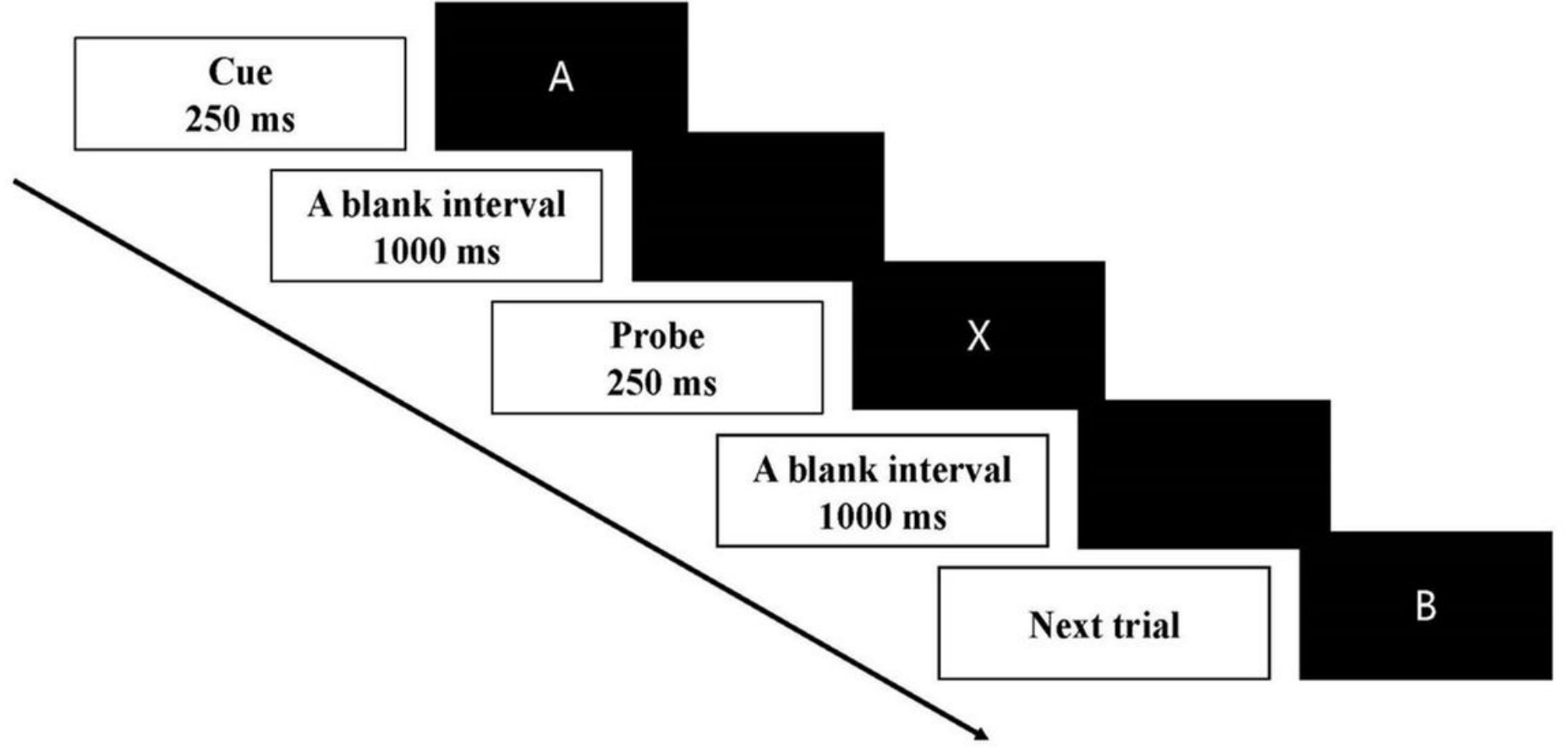

Figure 2

Schematic illustration of time course of one trial in AX-CPT.

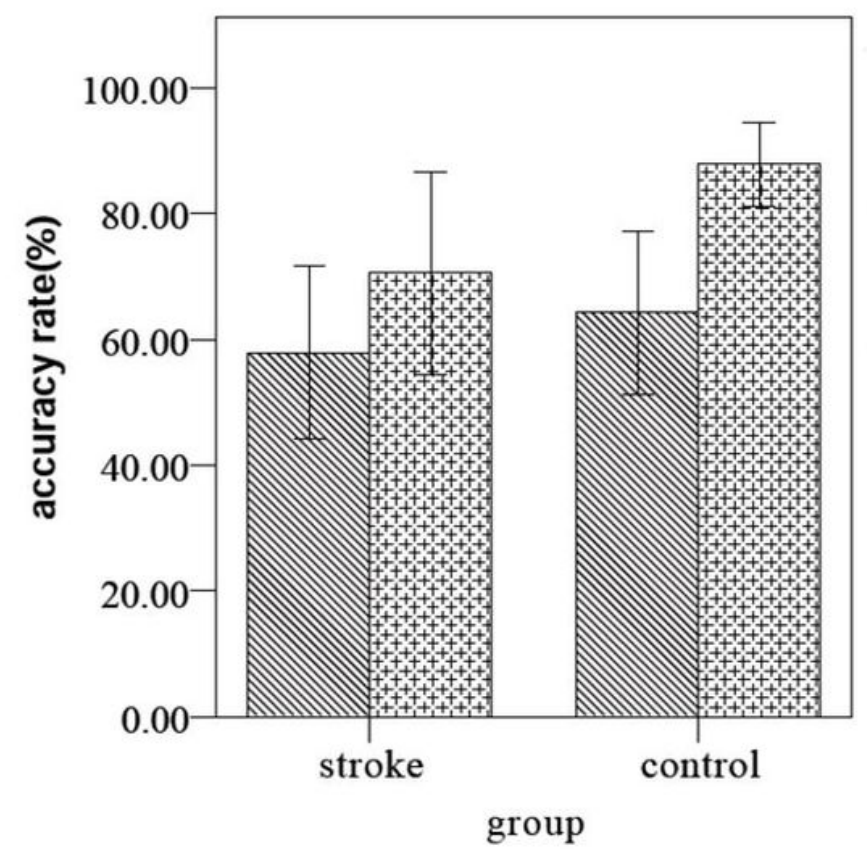

Error Bars: +1- $2 \mathrm{SE}$

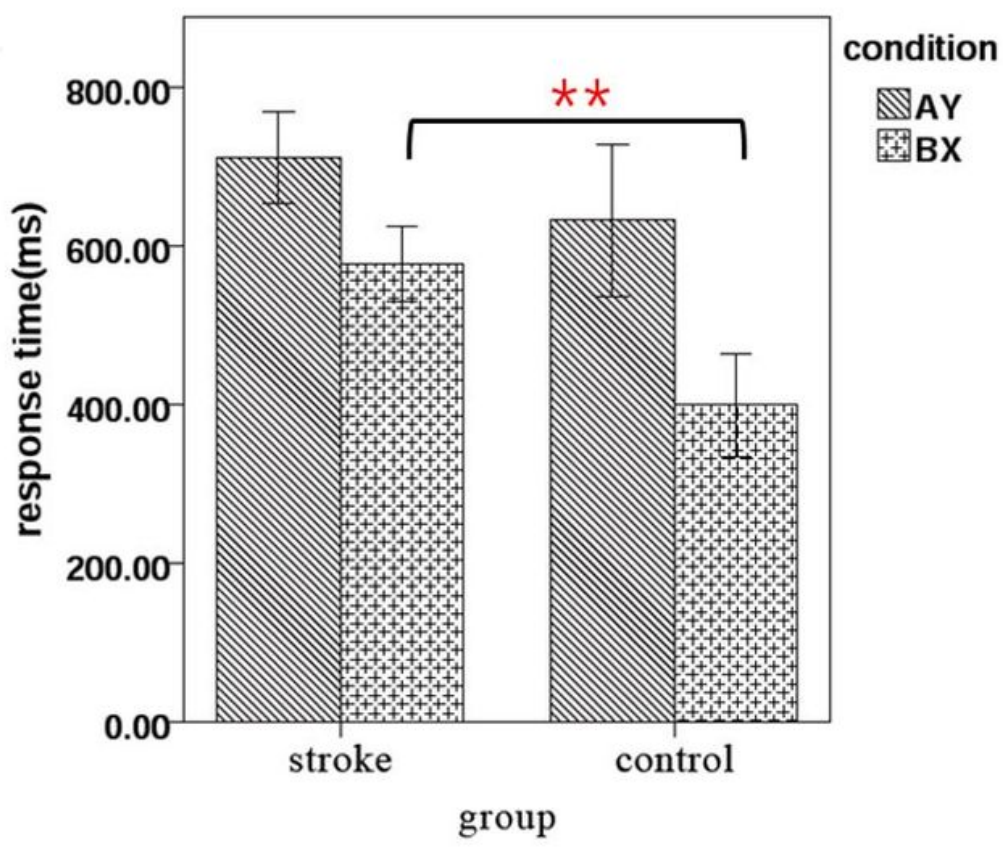

Error Bars: +/- 2 SE

Figure 3

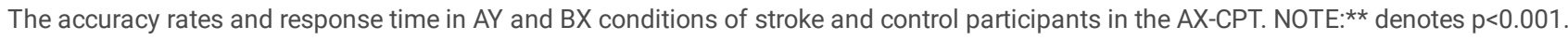

